\title{
ARTIGOS
}

\section{TERAPÊUTICA DA FASE CRÔNICA DA INFECÇÃO EXPERIMENTAL PELO TRYPANOSOMA CRUZI COM O BENZONIDAZOL E O NIFURTIMOX}

\author{
Sonia G. Andrade, Juracy B. Magalhães e Albélia L. Pontes
}

\begin{abstract}
Foram submetidos à quimioterapia com Nifurtimox (Bay 2502) ou com o Benzonidazol (Ro 7-1051) cinqüenta e oito camundongos cronicamente infectados com diferentes cepas do Trypanosoma cruzi (Tipos II e III) por periodos de 90 a 100 dias. Vinte e um camundongos cronicamente infectados foram utilizados como controles não tratados. Os inóculos variaram entre $1 \times 10^{4}$ e $5 \times 10^{4}$ tripomastigotas sanguicolas. $O$ tratamento foi feito durante 90 dias, nas doses de $200 \mathrm{mg} / \mathrm{kg} /$ dia durante 4 dias, seguidas de doses de $50 \mathrm{mg} / \mathrm{kg} /$ dia, para o Nifurtimox e de $100 \mathrm{mg} / \mathrm{kg} /$ dia, para o Benzonidazol. Os resultados dos testes parasitológicos (xenodiagnóstico, subinoculacão do sangue e hemocultura), nos camundongos tratados com o Benzonidazol, mostraram $85,3 \%$ de negativação nos animais infectados com as cepas de Tipo II e $43 \%$ com as cepas de Tipo III. Nos camundongos tratados com o Nifurtimox observou-se $71,4 \%$ de cura parasitológica nos infectados com as cepas de Tipo II e 66\% nos infectados com as cepas de Tipo III. Os testes de IFI permaneceram positivos em $90 \%$ dos animais tratados e curados. Houve regressão total ou parcial das lesões miocárdicas e de músculo esquelético nos animais tratados e curados. Em 50\% dos animais em que os testes de cura permaneceram positivos, houve persistência de lesões histopatológicas discretas. Conclui-se que o tratamento na fase crônica pode determinar negativação parasitológica em percentagem elevada de casos, comparável ao obtido na fase aguda, com as cepas de Tipo II e mais elevadas com as cepas de Tipo III, com persistência da positividade da IFI.
\end{abstract}

Palavras-chaves: Trypanosoma cruzi. Infecção crônica. Histopatologia. Quimioterapia. Benzonidazol. Nifurtimox.

Os experimentos sobre a quimioterapia da doença de Chagas revelaram, recentemente, alguns compostos eficazes no tratamento da fase aguda e da fase crônica da infecção experimental pelo $T$. cruzi, como o MK-436 $16^{18}$, o Megazol ${ }^{16}$ e o Cetoconazol $^{17}$. No entanto, o tratamento clínico de pacientes das áreas endêmicas continua a ser feito preferencial ou exclusivamente com Benzonidazol ( $\mathrm{N}$-benzyl-2nitro-1 imidazoleacetamida) ou com Nifurtimox (3-

Centro de Pesquisas Gonçalo Moniz/FIOCRUZ. Universidade Federal da Bahia.

Suporte financeiro: Conselho Nacional de Desenvolvimento Cientifico e Tecnológico ( $\mathrm{CNPq}$ ) e UNDP/WORLD BANK WHO Special Program for Research and Training in Tropical Diseases.

Endereço para correspondência: Rua Valdemar Falcão, 121 - Brotas, 41945 - Salvador, Bahia, Brasil.

Recebido para publicação em 21-3-89. metil-4 (5 nitrofurfurilideno amino) tetraidro $4 \mathrm{H}-1$, 4-tiazina-1, 1-dióxido). Ambos têm-se mostrado eficazes no tratamento da fase aguda, porém as tentativas de tratamento da fase crônica têm dado resultados pouco animadores 151920 . Torna-se, portanto, necessário, melhor avaliar a ação dos quimioterápicos sobre a infecção crônica pelo $T$. cruzi, considerando-se, inclusive, a ação do tratamento sobre as lesões fibrótico-inflamatórias peculiares à cardiopatia crônica chagásica, tanto no homem ${ }^{12}$, como no modelo murino 7 .

Há evidências de que o tratamento especifico com o Nifurtimox, mesmo em doses não curativas, pode levar a uma diminuição das lesões anatomopatológicas de camundongos cronicamente infecta$\operatorname{dos}^{2}$. Estudos recentes, utilizando a droga MK-4359, na infecção crônica experimental, mostraram a negativação parasitológica em elevada percentagem de casos e a regressão total ou parcial das lesões inflamatórias em miocárdio e músculo esquelético. 
Andrade SG, Magalhães $J B$, Pontes AL. Terapêutica da fase crônica da infecçāo experimental pelo Trypanosoma cruzi como benzonidazol e o nifurtimox. Revista da Sociedade Brasileira de Medicina Tropical 22: 113-118, Jul-Set, 1989

O presente trabalho tem por objetivo investigar a ação antiparasitária do Benzonidazol e Nifurtimox na fase crônica da infecção pelo $T$. cruzi, a possibilidade de cura parasitológica, a influência da cepa do parasito sobre esta resposta e a ação do tratamento sobre o quadro histopatológico.

\section{MATERIAL E MÉTODOS}

Foram utilizados 79 camundongos suiços na fase crônica de infecção pelo $T$. cruzi, os quais tinham sido inoculados com cepas virulentas e sobreviveram por periodos variáveis entre 90 e 400 dias. Os inóculos utilizados consistiam de formas sangüicolas, provenientes de camundongos infectados, variando entre $1 \mathrm{x}$ $10^{4}$ e $5 \times 10^{4}$ tripomastigotas, por via intraperitoneal. As cepas do $T$. cruzi utilizadas foram previamente caracterizadas biologicamente nos Tipos II e III de acordo com critérios já estabelecidos por Andrade ${ }^{1} \mathrm{e}$ recomendados pela $\mathrm{WHO}^{22}$ e foram as seguintes: cepas do Tipo II - 12 SF e 21 SF de São Felipe-BA; 13 MAM e 14 MAM, de Mambaí-GO; 1 COTEG e 2 COTEG de Cotegipe-BA, cepas de Tipo III 19 e 25 MONT, de Montalvânia-MG, cepa Bolívia-BO, Cepa Tinte (Chile).

Foram constituidos os seguintes grupos ( Tabela 1): a) 41 camundongos tratados com Benzonidazol, sendo 34 infectados com cepas de Tipo II e 7 com para subinoculação em camundongos recém-nascidos, hemocultura e obtenção de soro. A reação sorológica utilizada foi o teste de imunofluorescência indireta (IFI) de acordo com Camargo ${ }^{14}$. Os camundongos sacrificados foram submetidos a autópsias completas e após fixação em formol a $10 \%$ e inclusão em parafina, obtidas secções de $5 \mu \mathrm{m}$, coradas pelo hematoxilina e eosina, para estudo histopatológico. Para avaliação do grau de lesões em miocárdio e músculo esquelético fez-se uma graduação arbitrária: + indicando lesões discretas e focais, ++ lesões moderadas difusas ou focais e +++ lesões difusas e intensas.

\section{RESULTADOS}

A Tabela 2 mostra os indices de cura parasitológica dos camundongos tratados na fase crônica. No Tabela 2 - Indices de cura parasitológica em camundongos com infecção crônica pelo $\mathrm{T}$. cruzi, tratados com Benzonidazol e $\mathrm{Ni}$ furtimox.

\begin{tabular}{lcc}
\hline Cepas do & Índices de cura parasitológica \\
\cline { 2 - 3 } T. cruzi & Benzonidazol & Nifurtimox \\
\hline Tipos II & $85,3 \%$ & $71,4 \%$ \\
Tipo III & $43,0 \%$ & $66,0 \%$ \\
\hline
\end{tabular}

IFI Positivo em $100 \%$ dos tratados com Nifurtimox e em $81 \%$ dos tratados com Benzonidazole, curados parasitologicamente. Títulos de 10 a 320 .

Tabela 1 - Número de camundongos cronicamente infectados pelo T. cruzi (Cepas Tipo II e Tipo III) submetidos a quimioterapia com o Benzonidazol e o Nifurtimox.

\begin{tabular}{lcccc}
\hline Cepas do & Tratados com & Tratados com & Controles & Total \\
$T$. cruzi & Benzonidazol & Nifurtimox & não tratados & \\
\hline Tipo II & 34 & 14 & 15 & 63 \\
Tipo III & 7 & 3 & 6 & 16 \\
\hline Total & 41 & 17 & 21 & 79 \\
\hline
\end{tabular}

cepas de Tipo III; b) 17 camundongos tratados com Nifurtimox, sendo 14 infectados com cepas de Tipo II e 3 com cepas de Tipo III; c) 21 camundongoscontroles não tratados, sendo 15 infectados com cepas de Tipo II e 6 com cepas de Tipo III.

Esquemas terapêuticos: o Benzonidazol, composto nitroimidazólico (Ro 7-1051) foi administrado por entubação gástrica, na dose de $100 \mathrm{mg} / \mathrm{kg} / \mathrm{dia}(5$ doses semanais), durante 90 dias. O Nifurtimox, composto nitrofurânico (Bay 2502), foi administrado em 4 doses iniciais de $200 \mathrm{mg} / \mathrm{kg} / \mathrm{dia}$, seguidas por doses diárias (5 dias na semana) de $50 \mathrm{mg} / \mathrm{kg} / \mathrm{dia}$, durante 90 dias, por entubação gástrica. .

Testes de cura parasitológica: três e seis meses após o término do tratamento, os camundongos sobreviventes foram submetidos a xenodiagnóstico com ninfas do $3{ }^{\circ}$ estágio de $R$. prolixus e sacrificados sob anestesia pelo éter, sendo o sangue colhido utilizado grupo tratado com Benzonidazol, houve $85,3 \%$ de negativação parasitológica nos animais infectados com cepas de Tipo II e 43\% com as cepas de Tipo III. No grupo tratado com Nifurtimox, observou-se $71,4 \%$ de cura parasitológica nos infectados com cepas de Tipo II e 66\% nos infectados com cepas de Tipo III. Os testes sorológicos de IFI permaneceram positivos em $100 \%$ dos animais tratados com Nifurtimox e em $81 \%$ dos tratados com Benzonidazol e parasitologicamente curados com títulos variando de 10 e 320 .

Estudo histopatológico: os camundongos cronicamente infectados, mas não tratados (controles) apresentaram lesões inflamatórias e fibróticas de miocárdio e de músculo esquelético variando entre discretas e intensas (Tabela 3), (Figuras 1 A, B, C) e um processo de arterite necrotizante de grau variável (Figuras 1 C, D). 
Tabela 3 - Distribuição percentual dos casos tratados com Benzonidazol ou Nifurtimox de acordo com a intensidade das lesões histopatológicas em camundongos infectados com cepas de Tipo $I^{*}$.

\begin{tabular}{cccc}
\hline $\begin{array}{c}\text { Grau de } \\
\text { lesão }\end{array}$ & $\begin{array}{c}\text { Controles não } \\
\text { tratados }(\%)\end{array}$ & \multicolumn{2}{c}{ Tratados (\%) } \\
\cline { 3 - 4 } & & Benzonidazol & Nifurtimox \\
\hline Sem lesão & 0 & 20,8 & 57,1 \\
+ & 53,8 & 45,8 & 42,8 \\
++ & 38,4 & 20,8 & 0 \\
+++ & 7,6 & 0 & 0 \\
\hline
\end{tabular}

(*) Excluídos os animais parasitologicamente positivos após tratamento.
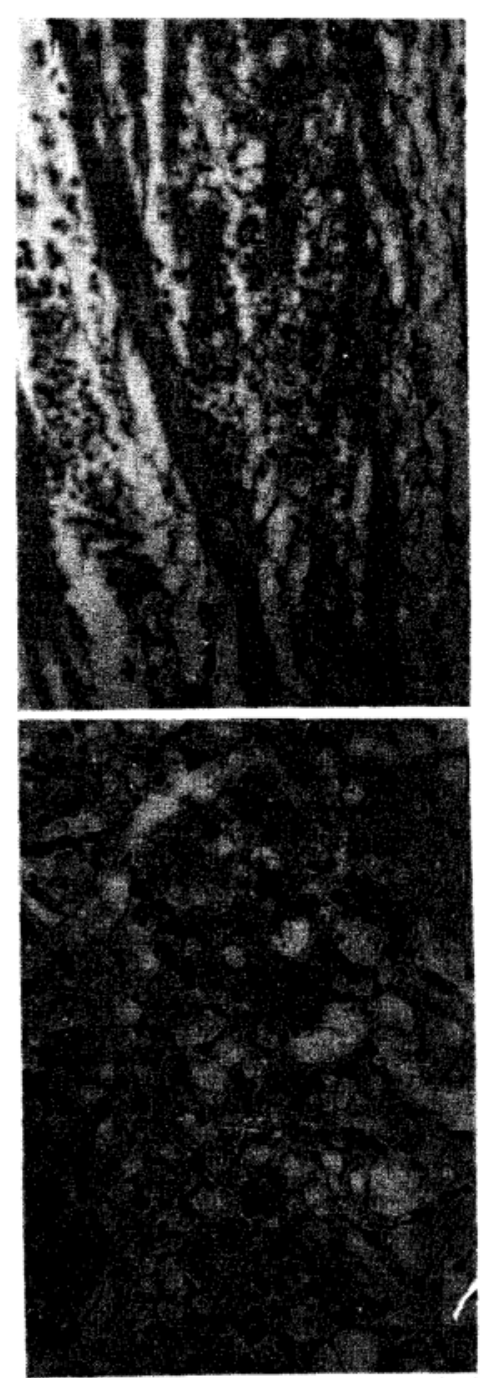

Figuras 1 A e 1 B - Miocardite crônica difusa em camundongos cronicamente infectados com cepa de Tipo II (12 SF) $100 x$ e $250 \times$ H.E.

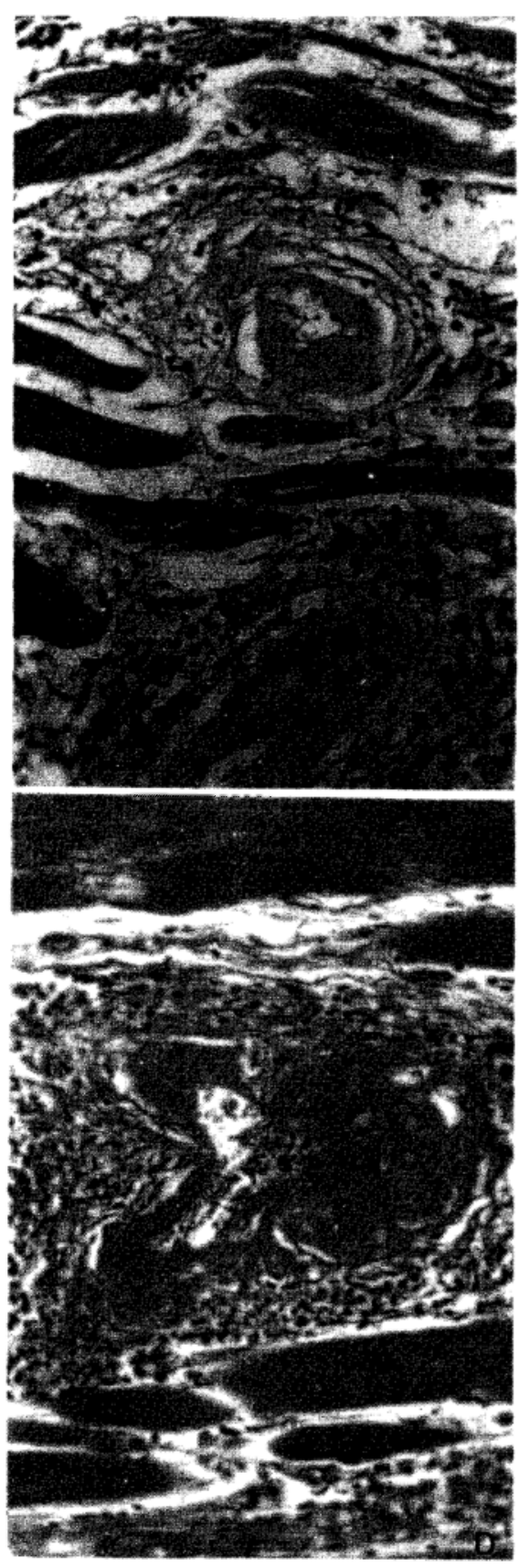

Figuras $1 \mathrm{C}$ e 1 D-Miosite crônica com áreas de destruicâo de fibras musculares esqueléticas e processo de arteriolite necrotizante em camundongos cronicamente infectados com cepa de Tipo III (Colombiana). Crontroles não tratados. $250 \times$ H.E.

Nos camundongos tratados com Benzonidazol ou com Nifurtimox e parasitologicamente curados, o miocárdio e o músculo esquelético não mostraram lesões histopatológicas em $20,8 \%$ dos tratados com Benzonidazol e em 57,1\% nos tratados com Nifurti- 
Andrade SG, Magalhães JB, Pontes AL. Terapêutica da fase crônica da infecçāo experimental pelo Trypanosoma cruzi como benzonidazol e o nifurtimox. Revista da Sociedade Brasileira de Medicina Tropical 22: 113-118, Jul-Set, 1989

mox. Nos demais casos as lesões eram discretas ou moderadas, com miocardite focal em áreas cicatriciais do miocárdio ou perivasculares e subepicárdicas (Figuras 2, A, B). Em $11 \%$ dos animais tratados com Benzonidazol havia um processo residual de arterite em miocárdio e em músculo esquelético (Figuras $2 \mathrm{C}$, D). $\mathrm{Na}$ Tabela 3 são apresentados dados relativos à distribuição percentual dos casos, de acordo com a intensidade das lesões histopatológicas. Nos camundongos tratados e não curados as lesões histopatológicas eram discretas ou moderadas, sendo intensas em apenas $1 / 10$ casos, estando ausentes em $4 / 10$ casos.
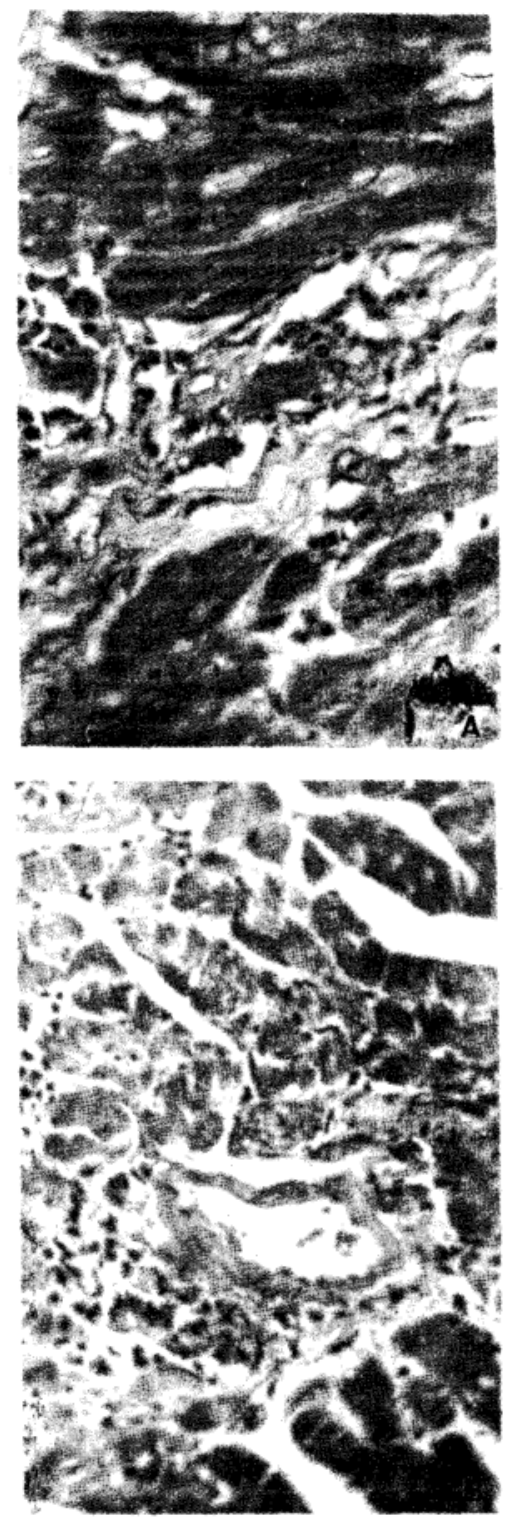

Figuras 2 A e 2 B-Miocardite residual em camundongos cronicamente infectados e tratados com Benzonidazol. $250 x$ e $100 x$ H.E.
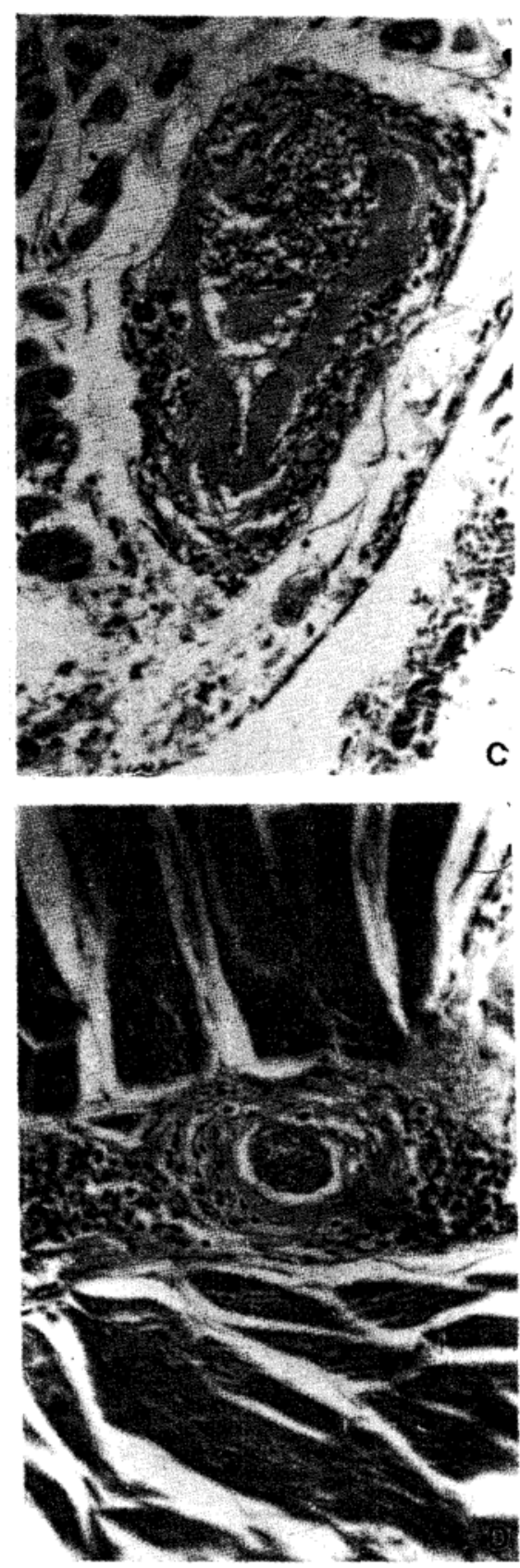

Figuras $2 \mathrm{C}$ e $2 \mathrm{D}$ - Processo residual de arterite em miocárdio e em músculo esquelético em camundongos cronicamente infectados e tratados com Nifurtimox. $100 x$ e 250 x H.E.

\section{DISCUSSÃO}

Os achados do presente trabalho indicam que na fase crônica da infecção pelo $T$. cruzi há uma resposta aos quimioterápicos de reconhecida ação antiparasitária, Nifurtimox e Benzonidazol, cuja ação destrutiva 
Andrade SG, Magalhães.JB, Pontes AL. Terapêutica da fase crônica da infecçāo experimental pelo Trypanosoma cruzi como benzonidazol e o nifurtimox. Revista da Sociedade Brasileira de Medicina Tropical 22: 113-118, Jul-Set, 1989

sobre as formas intracelulares do parasito in vivo já foi bem demonstrada ${ }^{35}$. Mesmo cepas que se mostraram altamente resistentes ao tratamento na fase aguda 348 responderam ao tratamento na infecção crônica do camundongo. Os indices de cura foram mesmo mais elevados nesta fase do que os anteriormente obtidos na fase aguda com as cepas de Tipo III cujo padrão é a cepa Colombiana. Observou-se, entretanto, maior susceptibilidade das cepas de Tipo II, com indices significativamente mais elevados de cura do que os obtidos com as cepas de Tipo III. Deve-se levar em conta, na análise destes dados, as dificuldades para se detectarem os parasitos aos exames parasitológicos, usuais, inerentes à fase crônica da infecção. Entretanto, os múltiplos testes parasitológicos realizados provavelmente teriam detectado a infecção, caso a mesma estivesse ainda presente. Por outro lado, a regressão das lesões histopatológicas é um dado muito importante para se avaliar a cura. Assim, nenhum animal-controle não tratados era isento de lesões, enquanto $20,8 \%$ dos tratados com Benzonidazol e $57,1 \%$ dos tratados com Nifurtimox e que estavam parasitologicamente negativos, não mostravam alterações histopatológicas. Nos animais curados em que persistiram lesões, estas eram discretas ou residuais. Além disto, mesmo nos animais que foram tratados, mas não estavam curados, raramente ocorreram lesões significantes.

As lesões de arterite necrotizante estavam presentes em uma percentagem de casos tratados, menos intensas do que nos animais não tratados e ocorriam mesmo na ausência de lesões difusas de miocardite ou miosite. Estas lesões têm sido atribuidas à presença de complexos imunes e, no caso dos animais tratados, poderiam talvez indicar a persistencia de antígenos parasitários.

Também no tratamento da infecção crônica pelo $T$. cruzi, com o MK-346, aspectos semelhantes de regressão de lesões histopatológicas e persistência de arterite necrotizante foram assinaladas 9 .

O processo inflamatório que ocorre na infecção crônica pelo $T$. cruzi tem a sua patogènese ligada a um processo de hipersensibilidade retardada ${ }^{11} 13$. Embora fenômenos de auto-imunidade possam estar envolvidos neste processo ${ }^{21}$, o fator parasito deve ser importante elemento na manutenção destas reações, e a sua eliminação poderá determinar a regressão do processo inflamatório.

Por outro lado, a persistência de positividade da reação sorológica de imunofluorescência indireta em animais aparentente curados pode estar relacionada com a presença de antígenos parasitários retidos em células dendriticas do baço, como recentemente demonstrado através da imunoeletromicroscopia ${ }^{6}$, confirmando a presença de uma "memória imunológica" capaz de manter o organismo em um processo de estimulação específica.

\section{SUMMARY}

Fifty-eight mice, chronically infected with different $\mathrm{T}$. cruzi strains (Types II and III) were submitted to chemotherapy either with Nifurtimox (Bay 2502) or Benznidazole (Ro 7-1051). Twenty one mice were not treated and were used as infected controls. The duration of infection was from 90 to 400 days. Inocula varied from $1 \times 10^{4}$ to $5 \times 10^{4}$ blood forms. Treatment lasted for 90 days, doses being $200 \mathrm{mg} / \mathrm{kg} /$ day during 4 days, followed by $50 \mathrm{mg} / \mathrm{kg} /$ day for Nifurtimox and $100 \mathrm{mg} / \mathrm{kg} /$ day for Benznidazole. Parasitological tests (xenodiagnosis, inoculations into baby mice and hemoculture) showed $85.3 \%$ negativation for Type II strains and 43\% for Type III in animals treated with Benznidazole. As for Nifurtimox, there were $71.4 \%$ of parasitological negativation for the animals infected with Type II strains and 66\% for those infected with Type III. IFA tests remained positive in $90 \%$ of treated and cured animals. Disappearance or marked regression of myocardial and skeletal muscle lesions was seen in the treated and parasitologically negative animals. The conclusion is that the treatment in the chronic phase of $\mathrm{T}$. cruzi infection can result in parasitological cure in a high percentage of cases with regression of histopathological lesions, although with persistence of positivity of the IFA tests.

Key-words: Trypanosoma cruzi. Chronic infection. Histopathology: Chemotherapy. Benznidazole. Nifurtimox.

\section{REFERÊNCIAS BIBLIOGRÁFICAS}

1. Andrade SG. Caracterização de cepas do Trypanosoma cruzi isoladas no Recôncavo baiano. Revista de Patologia Tropical 3:65-121, 1974.

2. Andrade SG, Andrade ZA. Aspectos anatomopatológicos e resposta terapêutica na infecção chagásica crônica experimental. Revista do Instituto de Medicina Tropical de São Paulo 18:268-275, 1976.

3. Andrade SG, Figueira RM. Estudo experimental sobre a ação terapêutica da droga Ro 7-1051 na infecção por diferentes cepas do Trypanosoma cruzi. Revista do Instituto de Medicina Tropical de São Paulo 19:335-341, 1977.

4. Andrade SG, Figueira RM, Carvalho ML, Gorini DF. Influência da cepa do Trypanosoma cruzi na resposta à terapêutica e experimental pelo Bay 2502. Resultados de tratamento a longo prazo. Revista do Instituto de Medicina Tropical de São Paulo 17:380-389, 1975.

5. Andrade SG, Freitas LAR. Trypanosoma cruzi: cardiac myocell alterations due to spontaneous or therapeutically induced intracellular parasite desintegration. Cellular and Molecular Biology 33:797-805, 1987. 
6. Andrade SG, Freitas LAR, Peyrol, S, Pimentel AR, Sadigursky M. Trypanosoma cruzi antigens detected by immunoelectron microscopy in the spleen of mice serologically positive but parasitologically cured by chemotherapy. Preliminary report. Revista da Sociedade Brasileira de Medicina Tropical 21:41-42, 1988.

7. Andrade SG, Grimaud JA. Chronic murine myocarditis due to Trypanosoma cruzi: an ultrastructural study and immunochemical characterization of cardiac interstitial matrix. Memórias do Instituto Oswaldo Cruz 81:29-41, 1986.

8. Andrade SG, Magalhāes JB, Pontes AL. Evaluation of chemotherapy with benznidazole and nifurtimox in mice infected with Trypanosoma cruzi strains of different types. Bulletin of the World Health Organization 63:721-726, 1985.

9. Andrade SG, Silva RC, Santiago CMG. Treatment of chronic Trypanosoma cruz $i$ experimental infection vith MK-436 (2-5-nitroimidazole). Bulletin of the World Health Organization 67: 509-514, 1989.

10. Andrade SG, Silva RC, Santiago CMG, Freitas LAR. Therapeutic action of MK-436 (2-5-nitroimidazole) on Trypanosoma cruzi infections in mice: a parasitological, serological, histopathological and ultrastructural study. Bulletin of the World Health Organization 65:625-633, 1987.

11. Andrade ZA. Mechanisms of myocardial damage in Trypanosoma cruzi infection. In: Cytopathology of parasitic diseases. (CIBA Foundation Symposium, 99), London, Pitman Books, p.214-233, 1983.

12. Andrade ZA, Andrade SG. Patologia. In: Brener Z, Andrade $\mathrm{Z}$ (ed): Trypanosoma cruzi e doença de Chagas. Rio de Janeiro, Guanabara Koogan, p.199-248, 1979.

13. Andrade ZA, Andrade SG, Sadigursky M. Enhancement of chronic Trypanosoma cruzi myocarditis in dogs trea- ted with low doses of cyclophosphamide. American Journal of Pathology 127: 467-473, 1987.

14. Camargo ME. Fluorescent antibody test for the serodiagnosis of American trypanosomiasis. Technical modification employing preserved culture forms of Trypanosoma cruzi in a slide test. Revista do Instituto de Medicina Tropical de São Paulo 8:227-234, 1966.

15. Cançado JR. Tratamento específico. In: Cançado JR Chuster M. (ed): Cardiopatia chagásica, Fundação Carlos Chagas, Belo Horizonte p.327-355, 1985.

16. Filardi LS, Brener Z. A nitroimidazole-thiadiazole derivative with curative action in experimental Trypanosoma cruzi infections. Annals of Tropical Medicine and Parasitology 76:293-297, 1982.

17. McCabe RE, Remington JS, Araujo FG. Ketoconazole promotes parasitological cure of mice infected with Trypanosoma cruzi. Transactions of the Royal Society of Tropical Medicine and Hygiene 81:613-615, 1987.

18. Murray PK, Habbersett MC, Meurer RD. Trypanosoma cruzi: efficacy of the 2-substituted 5-nitroimidazoles, MK-436 and L 634, 549, in tissue culture and mice. American Journal of Tropical Medicine and Hygiene 32:1242-1250, 1983 .

19. Rassi A. Tratamento etiológico da doença de Chagas. Arquivos Brasileiros de Cardiologia 39:277-281, 1982.

20. Rassi A, Ferreira HO. Tentativas de tratamento especifico da fase aguda da doença de Chagas com nitrofuranos em esquemas de duração prolongada. Revista da Sociedade Brasileira de Medicina Tropical 5:235-262, 1971.

21. Sadigursky M, Acosta AM, Santos-Buch A Muscle sarcoplasmic reticulum antigen shared by a Trypanosoma cruzi clone. American Journal of Tropical Medicine and Hygiene 31:934-941, 1982.

22. WHO - Research Activities of the Scientific Working Group (SWG) on Chagas'disease - 1982-1985. Memórias do Instituto Oswaldo Cruz 81 (Suppl.): 179-244, 1986. 